\title{
Critical Oscillation Constant for Euler Type Half-Linear Differential Equation Having Multi-Different Periodic Coefficients
}

\author{
Adil Misir and Banu Mermerkaya \\ Department of Mathematics, Faculty of Science, Gazi University, Teknikokullar, 06500 Ankara, Turkey \\ Correspondence should be addressed to Adil Misir; adilm@gazi.edu.tr
}

Received 15 July 2016; Accepted 12 October 2016; Published 27 February 2017

Academic Editor: Said R. Grace

Copyright (C) 2017 Adil Misir and Banu Mermerkaya. This is an open access article distributed under the Creative Commons Attribution License, which permits unrestricted use, distribution, and reproduction in any medium, provided the original work is properly cited.

We compute explicitly the oscillation constant for Euler type half-linear second-order differential equation having multi-different periodic coefficients.

\section{Introduction}

In literature, half-linear second-order differential equations are given by

$$
\begin{aligned}
\left(r(t) \Phi\left(x^{\prime}\right)\right)^{\prime}+c(t) \Phi(x) & =0, \\
\Phi(s) & =|s|^{p-2} s, \quad p>1,
\end{aligned}
$$

where $r, c$ are continuous functions and $r(t)>0$. It is well known that oscillation theory of (1) is very similar to that of the linear Sturm-Liouville differential equation, which is the special case of $p=2$ in (1); see [1].

In particular, (1) with $\lambda c(t)$ instead of $c(t)$ is said to be conditionally oscillatory if there exists a constant $\lambda_{0}$ such that this equation is oscillatory for $\lambda>\lambda_{0}$ and nonoscillatory for $\lambda<\lambda_{0} . \lambda_{0}$ is called the critical oscillation constant of this equation; see [2].

The half-linear Euler differential equation

$$
\left(\Phi\left(x^{\prime}\right)\right)^{\prime}+\frac{\gamma_{p}}{t^{p}} \Phi(x)=0
$$

with the so-called critical oscillation constant $\gamma_{p}=((p-$ $1) / p)^{p}$, plays an important role in the conditionally oscillatory half-linear differential equation.

Equation (2) can be regarded as a good comparative equation in the sense that (2) with $\gamma$ instead of $\gamma_{p}$ is oscillatory if and only if $\gamma>\gamma_{p}$ (see [3]) and if $r(t)=1$ in (1), then this equation is oscillatory provided

$$
\lim _{t \rightarrow \infty} \inf t^{p} c(t)>\gamma_{p}
$$

and nonoscillatory if

$$
\lim _{t \rightarrow \infty} \sup t^{p} c(t)<\gamma_{p}
$$

see $[4]$.

In [5], perturbations of (2) being of the form

$$
\left(\Phi\left(x^{\prime}\right)\right)^{\prime}+\frac{1}{t^{p}}\left(\gamma_{p}+\sum_{l=1}^{n} \frac{\beta_{j}}{\log _{j}^{2} t}\right) \Phi(x)=0
$$

are investigated when $\lim _{t \rightarrow \infty} t^{p} c(t)=\gamma_{p}$ for constant $\beta_{j}(j=$ $1,2, \ldots, n)$. Here the notation

$$
\begin{aligned}
\log _{k} t & =\prod_{j=1}^{k} \log _{j} t, \\
\log _{k} t & =\log _{k-1}(\log t), \\
\log _{1} t & =\log t
\end{aligned}
$$

is used. It is shown that the constant $\mu_{p}:=(1 / 2)((p-1) / p)^{p-1}$ plays a crucial role in (5). In particular, if $n=1$ in (5) this 
equation reduces to the so-called Riemann-Weber half-linear differential equation, and this equation is oscillatory if $\beta_{1}>$ $\mu_{p}$ and nonoscillatory otherwise. In general, if $\beta_{j}=\mu_{p}$ for $j=1,2, \ldots, n-1$, then (5) is oscillatory if and only if $\beta_{n}>\mu_{p}$.

One of the typical problems in the qualitative theory of various differential equations is to study what happens when constants in an equation are replaced by periodic functions which have same periods and different periods. Our investigation follows this line and it is mainly motivated by the paper [6]. form

In [7], the half-linear differential equation being of the

$$
\begin{aligned}
\left(\left(1+\sum_{j=1}^{n} \frac{\alpha_{j}}{\log _{j}^{2} t}\right)^{1-p} \Phi\left(x^{\prime}\right)\right)^{\prime} \\
+\frac{1}{t^{p}}\left(\gamma_{p}+\sum_{j=1}^{n} \frac{\beta_{j}}{\log _{j}^{2} t}\right) \Phi(x)=0
\end{aligned}
$$

is investigated for $\alpha_{j}$ and $\beta_{j}$ are constants and the following result is obtained.

Theorem 1. Suppose that there exists $k \in\{2, \ldots, n\}$ such that

$$
\beta_{j}+(p-1) \gamma_{p} \alpha_{j}=\mu_{p}, \quad j=1, \ldots, k-1
$$

and $\beta_{k}+(p-1) \gamma_{p} \alpha_{k} \neq \mu_{p}$. Then (7) is oscillatory if $\beta_{k}+(p-$ 1) $\gamma_{p} \alpha_{k}>\mu_{p}$ and nonoscillatory if $\beta_{k}+(p-1) \gamma_{p} \alpha_{k}<\mu_{p}$.

In [8], the half-linear differential equation being of the form

$$
\left(r(t) \Phi\left(x^{\prime}\right)\right)^{\prime}+\frac{\gamma c(t)}{t^{p}} \Phi(x)=0
$$

is considered for $\alpha$-periodic positive functions $r$ and $c$ and it is shown that (9) is oscillatory if $\gamma>K$ and nonoscillatory if $\gamma<K$, where $K$ is given by

$$
K=q^{-p}\left(\frac{1}{\alpha} \int_{0}^{\alpha} \frac{d \tau}{r^{q-1}}\right)^{1-p}\left(\frac{1}{\alpha} \int_{0}^{\alpha} c(\tau) d \tau\right)^{-1}
$$

for $p$ and $q$ are conjugate numbers; that is, $1 / p+1 / q=1$.

In [9], (9) and the half-linear differential equation being of the form

$$
\left(r(t) \Phi\left(x^{\prime}\right)\right)^{\prime}+\frac{1}{t^{p}}\left(\gamma c(t)+\frac{\mu d(t)}{\log ^{2} t}\right) \Phi(x)=0
$$

are considered for $r, c$, and $d$ are $\alpha$-periodic, positive functions defined on $[0, \infty)$ and it is shown that (9) is nonoscillatory if and only if $\gamma \leq \gamma_{r c}$, where $\gamma_{r c}$ is given by

$$
\gamma_{r c}:=\frac{\alpha^{p} \gamma_{p}}{\left(\int_{0}^{\alpha} r^{1-q}(t) d t\right)^{p-1} \int_{0}^{\alpha} c(t) d t} .
$$

In the limiting case $\gamma=\gamma_{r c}$ (11) is nonoscillatory if $\mu<\mu_{r d}$ and it is oscillatory if $\mu>\mu_{r d}$, where $\mu_{r d}$ is given by

$$
\mu_{r d}=\frac{\alpha^{p} \mu_{p}}{\left(\int_{0}^{\alpha} r^{1-q}(t) d t\right)^{p-1} \int_{0}^{\alpha} d(t) d t} .
$$

In [10], the half-linear differential equation being of the form

$$
\left(r(t) \Phi\left(x^{\prime}\right)\right)^{\prime}+\frac{c(t)}{t^{p}} \Phi(x)=0
$$

is considered for $r:[a, \infty) \rightarrow \mathbb{R},(a>0)$, is a continuous function for which mean value $M\left(r^{1-q}\right)$ exists and for which

$$
0<\inf _{t \in[a, \infty)} r(t) \leq \sup _{t \in[a, \infty)} r(t)<\infty
$$

holds and $c:[a, \infty) \rightarrow \mathbb{R},(a>0)$, is a continuous function having mean value $M(c)$ and it was shown that (14) is oscillatory if $M(c)>\Gamma$ and nonoscillatory if $M(c)<\Gamma$, where $\Gamma$ is given by

$$
\Gamma=q^{-p}\left[M\left(r^{1-q}\right)\right]^{1-p} .
$$
form

In [6], the half-linear differential equation being of the

$$
\begin{aligned}
& \left(\left(r(t)+\sum_{j=1}^{n} \frac{\alpha_{j}(t)}{\log _{j}^{2} t}\right)^{1-p} \Phi\left(x^{\prime}\right)\right)^{\prime} \\
& +\frac{1}{t^{p}}\left(c(t)+\sum_{j=1}^{n} \frac{\beta_{j}(t)}{\log _{j}^{2} t}\right) \Phi(x)=0
\end{aligned}
$$

is considered for $T$-periodic functions $r, c, \alpha_{j}$, and $\beta_{j}, j=$ $1,2, \ldots, n$, and $r(t)>0$ and the following result was obtained.

Theorem 2. Let $r, c, \alpha_{j}$, and $\beta_{j}(j=1,2, \ldots, n)$ be T-periodic continuous functions, $r(t)>0$, and their mean values over the period $T$ are denoted by $\widetilde{r}, \widetilde{c}, \widetilde{\alpha}_{j}$, and $\widetilde{\beta}_{j}(j=1,2, \ldots, n)$.

(i) If $\widetilde{c} \widetilde{r}^{p-1}>\gamma_{p}$, then (17) is oscillatory and if $\widetilde{c} \widetilde{r}^{p-1}<\gamma_{p}$, then it is nonoscillatory.

(ii) Let $\tilde{c} \widetilde{r}^{p-1}=\gamma_{p}$. If there exists $k \in\{1, \ldots, n\}$ such that

$$
\widetilde{\beta}_{j} \widetilde{r}^{p-1}+(p-1) \gamma_{p} \widetilde{\alpha}_{j} \widetilde{r}^{-1}=\mu_{p}, \quad j=1,2, \ldots, k-1
$$

(if $k \neq 1$ ), and $\widetilde{\beta}_{k} \widetilde{r}^{p-1}+(p-1) \gamma_{p} \alpha_{k} \widetilde{r}^{-1} \neq \mu_{p}$, then (17) is oscillatory if

$$
\widetilde{\beta}_{k} \widetilde{r}^{p-1}+(p-1) \gamma_{p} \widetilde{\alpha}_{k} \widetilde{r}^{-1}>\mu_{p}
$$

and nonoscillatory if

$$
\widetilde{\beta}_{k} \widetilde{r}^{p-1}+(p-1) \gamma_{p} \widetilde{\alpha}_{k} \widetilde{r}^{-1}<\mu_{p} .
$$

Our research is motivated by the paper [6], where the oscillation constant is computed for (17) with the periodic coefficients having same $T$-period. However, if these periodic functions have different periods what would be the oscillation constant is not investigated. Thus, in this paper we investigate the oscillation constant for (17) with periodic coefficients having different periods. In this paper we consider two types of periodic coefficients which have different periods 
for (17). In the first type we consider these periodic coefficient functions having the least common multiple and in the second type, we consider these periodic coefficient functions which do not have least common multiple. We give some corollaries which illustrate the first type's cases that our results compile the known results in [6] but in the second type only our results can be applied.

In Section 2, we recall the concept of half-linear-trigonometric functions and their properties. In Section 3 we compute the oscillation constant for (17) with periodic coefficients which have different periods. Additionally we show that if the different periods coincide, then our results compile with the known results in [6]. Thus, our results extend and improve the results of [6].

\section{Preliminaries}

We start this section with recalling the concept of half-lineartrigonometric functions; see [1] or [4]. Consider the following special half-linear equation being of the form

$$
\left(\Phi\left(x^{\prime}\right)\right)^{\prime}+(p-1) \Phi(x)=0
$$

and denote its solution by $x=x(t)$ given by the initial conditions $x(0)=0, x^{\prime}(0)=1$. We see that the behavior of this solution is very similar to that of the classical sine function. We denote this solution by $\sin _{p} t$ and its derivative by $\left(\sin _{p} t\right)^{\prime}=\cos _{p} t$. These functions are $2 \pi_{p}$-periodic, where $\pi_{p}:=2 \pi / p \sin (\pi / p)$, and satisfy the half-linear Pythagorean identity

$$
\left|\sin _{p} t\right|^{p}+\left|\cos _{p} t\right|^{p}=1, \quad t \in \mathbb{R} .
$$

Every solution of (21) is of the form $x(t)=C \sin _{p}(t+\varphi)$, where $C$ and $\varphi$ are real constants; that is, it is bounded together with its derivative and periodic with the period $2 \pi_{p}$. The function $u=\Phi\left(\cos _{p} t\right)$ is a solution to the reciprocal equation of (21);

$$
\begin{aligned}
& \left(\Phi^{-1}\left(u^{\prime}\right)\right)^{\prime}+(p-1)^{q-1} \Phi^{-1}(u)=0 \\
& \Phi^{-1}(u)=|u|^{q-2} u, q=\frac{p}{p-1}
\end{aligned}
$$

which is an equation of the form as in (21), so the functions $u$ and $u^{\prime}$ are also bounded.

Let $x(t)$ be a nontrivial solution of (1) and we consider the half-linear Prüfer transformation which is introduced using the half-linear-trigonometric functions

$$
\begin{aligned}
x(t) & =\rho(t) \sin _{p} \varphi(t), \\
x^{\prime}(t) & =r^{1-q}(t) \frac{\rho(t)}{t} \cos _{p} \varphi(t),
\end{aligned}
$$

where $\rho(t)=\sqrt{|x(t)|^{p}+r^{q}(t)\left|x^{\prime}(t)\right|^{p}}$ and Prüfer angle $\varphi(t)$ is a continuous function defined at all points where $x(t) \neq 0$.
Then $\varphi(t)$ satisfies the following differential equation:

$$
\begin{aligned}
\varphi^{\prime}(t) & =\frac{1}{t}\left[r^{1-q}(t)\left|\cos _{p} \varphi(t)\right|^{p}\right. \\
& \left.-\Phi\left(\cos _{p} \varphi(t)\right) \sin _{p} \varphi(t)+\frac{t^{p} c(t)}{p-1}\left|\sin _{p} \varphi(t)\right|^{p}\right] ;
\end{aligned}
$$

see [9].

\section{Main Results}

We need the following lemma in order to prove our main Theorem 4.

Lemma 3. Let $\varphi(t)=\varphi_{1}(t)+\sum_{j=1}^{n} \varphi_{2_{j}}(t)+\varphi_{3}(t)+\varphi_{4}(t)+$ $\sum_{j=1}^{n} \varphi_{5_{j}}(t)+M(M$ is a suitable constant) be a solution of the equation

$$
\begin{aligned}
\varphi^{\prime}(t)= & \varphi_{1}^{\prime}(t)+\sum_{j=1}^{n} \varphi_{2_{j}}^{\prime}(t)+\varphi_{3}^{\prime}(t)+\varphi_{4}^{\prime}(t) \\
& +\sum_{j=1}^{n} \varphi_{5_{j}}^{\prime}(t)
\end{aligned}
$$

where

$$
\begin{aligned}
\varphi_{1}^{\prime}(t) & =\frac{1}{t} r(t)\left|\cos _{p} \varphi(t)\right|^{p}, \\
\varphi_{2_{j}}^{\prime}(t) & =\frac{\alpha_{j}(t)}{t \log _{j}^{2} t}\left|\cos _{p} \varphi(t)\right|^{p} \quad(j=1, \ldots, n), \\
\varphi_{3}^{\prime}(t) & =-\frac{1}{t} \Phi\left(\cos _{p} \varphi(t)\right) \sin _{p} \varphi(t), \\
\varphi_{4}^{\prime}(t) & =\frac{c(t)}{(p-1) t}\left|\sin _{p} \varphi(t)\right|^{p}, \\
\varphi_{5_{j}}^{\prime}(t) & =\frac{\beta_{j}(t)}{(p-1) t \log _{j}^{2} t}\left|\sin _{p} \varphi(t)\right|^{p} \quad(j=1, \ldots, n),
\end{aligned}
$$

with $r, c, \alpha_{j}$, and $\beta_{j}(j=1,2, \ldots, n)$ are periodic functions having different $T_{1}, T_{2}, P_{j}$, and $Q_{j}(j=1,2, \ldots, n)$ periods, respectively, and $r(t)>0$ and

$$
\begin{aligned}
\theta(t)= & \frac{1}{T_{1}} \int_{t}^{t+T_{1}} \varphi_{1}(s) d s+\sum_{j=1}^{n} \frac{1}{P_{j}} \int_{t}^{t+P_{j}} \varphi_{2_{j}}(s) d s \\
& +\frac{1}{\xi} \int_{t}^{t+\xi} \varphi_{3}(s) d s+\frac{1}{T_{2}} \int_{t}^{t+T_{2}} \varphi_{4}(s) d s \\
& +\sum_{j=1}^{n} \frac{1}{Q_{j}} \int_{t}^{t+Q_{j}} \varphi_{5_{j}}(s) d s,
\end{aligned}
$$


where $\xi$ is one of the following $T_{1}, T_{2}, P_{j}$, and $Q_{j}(j=$ $1,2, \ldots, n)$ periods. Then $\theta(t)$ is a solution of

$\theta^{\prime}(t)$

$$
\begin{aligned}
= & \frac{1}{t}\left[\stackrel{*}{r}+\sum_{j=1}^{n} \frac{\dot{\alpha}_{j}^{*}}{\log _{j}^{2} t}+\frac{o(1)}{\log _{n}^{2} t}\right]\left|\cos _{p} \theta(t)\right|^{p} \\
& +\frac{1}{(p-1) t}\left[\stackrel{*}{c}+\sum_{j=1}^{n} \frac{\dot{\beta}_{j}^{*}}{\log _{j}^{2} t}+\frac{o(1)}{\log _{n}^{2} t}\right]\left|\sin _{p} \theta(t)\right|^{p} \\
& -\frac{1}{t} \Phi\left(\cos _{p} \theta(t)\right) \sin _{p} \theta(t),
\end{aligned}
$$

$$
\text { for } j=1,2, \ldots, n
$$

and $\varphi(\tau)-\theta(t)=\circ(1)$ as $t \rightarrow \infty$.

Proof. Taking derivative of $\theta(t)$, we have

$$
\begin{aligned}
\theta^{\prime}(t)= & \frac{1}{T_{1}} \int_{t}^{t+T_{1}} \varphi_{1}^{\prime}(s) d s+\sum_{j=1}^{n} \frac{1}{P_{j}} \int_{t}^{t+P_{j}} \varphi_{2_{j}}^{\prime}(s) d s \\
& +\frac{1}{\xi} \int_{t}^{t+\xi} \varphi_{3}^{\prime}(s) d s+\frac{1}{T_{2}} \int_{t}^{t+T_{2}} \varphi_{4}^{\prime}(s) d s \\
& +\sum_{j=1}^{n} \frac{1}{Q_{j}} \int_{t}^{t+Q_{j}} \varphi_{5_{j}}^{\prime}(s) d s \\
= & \frac{1}{T_{1}} \int_{t}^{t+T_{1}} \frac{1}{s} r(s)\left|\cos _{p} \varphi(s)\right|^{p} d s \\
& +\sum_{j=1}^{n} \frac{1}{P_{j}} \int_{t}^{t+P_{j}} \frac{\alpha_{j}(s)}{s \log _{j}^{2} s}\left|\cos _{p} \varphi(s)\right|^{p} d s \\
& -\frac{1}{\xi} \int_{t}^{t+\xi} \frac{1}{s} \Phi\left(\cos _{p} \varphi(s)\right) \sin _{p} \varphi(s) d s \\
& +\frac{1}{T_{2}} \int_{t}^{t+T_{2}} \frac{c(s)}{(p-1) s}\left|\sin _{p} \varphi(s)\right|^{p} d s \\
& +\sum_{j=1}^{n} \frac{1}{Q_{j}} \int_{t}^{t+Q_{j}} \frac{\beta_{j}(s)}{s(p-1) \log _{j}^{2} s}\left|\sin _{p} \varphi(s)\right|^{p} d s .
\end{aligned}
$$

Using integration by parts, we get

$$
\begin{aligned}
& \theta^{\prime}(t)=\frac{1}{T_{1} t} \int_{t}^{t+T_{1}} r(\tau)\left|\cos _{p} \varphi(\tau)\right|^{p} d \tau+\frac{1}{t} \sum_{j=1}^{n} \frac{1}{P_{j}} \\
& \cdot \int_{t}^{t+P_{j}} \frac{\alpha_{j}(\tau)}{\log _{j}^{2} \tau}\left|\cos _{p} \varphi(\tau)\right|^{p} d \tau-\frac{1}{\xi t} \\
& \cdot \int_{t}^{t+\xi} \Phi\left(\cos _{p} \varphi(\tau)\right) \sin _{p} \varphi(\tau) d \tau+\frac{1}{T_{2} t} \\
& \cdot \int_{t}^{t+T_{2}} \frac{c(\tau)}{(p-1)}\left|\sin _{p} \varphi(\tau)\right|^{p} d \tau+\frac{1}{t} \sum_{j=1}^{n} \frac{1}{Q_{j}} \\
& \cdot \int_{t}^{t+Q_{j}} \frac{\beta_{j}(\tau)}{(p-1) \log _{j}^{2} \tau}\left|\sin _{p} \varphi(\tau)\right|^{p} d \tau-\frac{1}{T_{1}} \\
& \cdot \int_{t}^{t+T_{1}} \frac{1}{s^{2}} \int_{s}^{t+T_{1}} r(\tau)\left|\cos _{p} \varphi(\tau)\right|^{p} d \tau d s-\sum_{j=1}^{n} \frac{1}{P_{j}} \\
& \cdot \int_{t}^{t+P_{j}} \frac{1}{s^{2}} \int_{t}^{t+P_{j}} \frac{\alpha_{j}(\tau)}{\log _{j}^{2} \tau}\left|\cos _{p} \varphi(\tau)\right|^{p} d \tau d s+\frac{1}{\xi} \\
& \cdot \int_{t}^{t+\xi} \frac{1}{s^{2}} \int_{s}^{t+\xi} \Phi\left(\cos _{p} \varphi(\tau)\right) \sin _{p} \varphi(\tau) d \tau d s-\frac{1}{T_{2}} \\
& \cdot \int_{t}^{t+T_{2}} \frac{1}{s^{2}} \int_{s}^{t+T_{2}} \frac{c(\tau)}{(p-1)}\left|\sin _{p} \varphi(\tau)\right|^{p} d \tau d s \\
& -\sum_{j=1}^{n} \frac{1}{Q_{j}} \\
& \cdot \int_{t}^{t+Q_{j}} \frac{1}{s^{2}} \int_{t}^{t+Q_{j}} \frac{\beta_{j}(\tau)}{(p-1) \log _{j}^{2} \tau}\left|\sin _{p} \varphi(\tau)\right|^{p} d \tau d s .
\end{aligned}
$$

Let $f$ be a continuous $T$-periodic function and ${ }^{*}=$ $(1 / T) \int_{0}^{T} f(s) d s$; then integration by parts yields

$$
\frac{1}{T} \int_{t}^{t+T} \frac{f(s)}{\log _{j}^{2} s} d s=\frac{\stackrel{f}{f}}{\log _{j}^{2} t}\left[1+O\left(\frac{1}{t \log t}\right)\right] .
$$

By using (33) and $\int_{t}^{t+T} f(s) d s=\int_{0}^{T} f(s) d s$ for any $T$-periodic function and Pythagorean identity, the expressions

$$
\begin{gathered}
r^{1-q}(t)\left|\cos _{p} \varphi(t)\right|^{p}, \\
-\Phi\left(\cos _{p} \varphi(t)\right) \sin _{p} \varphi(t), \\
\frac{c(t)}{p-1}\left|\sin _{p} \varphi(t)\right|^{p}
\end{gathered}
$$

are bounded. Thus we get

$\theta^{\prime}(t)$

$$
=\frac{1}{T_{1} t} \int_{t}^{t+T} r(\tau)\left|\cos _{p} \varphi(\tau)\right|^{p} d \tau
$$




$$
\begin{aligned}
& +\frac{1}{t} \sum_{j=1}^{n} \frac{1}{P_{j}} \int_{t}^{t+P_{j}} \frac{\alpha_{j}(\tau)}{\log _{j}^{2} \tau}\left|\cos _{p} \varphi(\tau)\right|^{p} d \tau \\
& -\frac{1}{\xi t} \int_{t}^{t+\xi} \Phi\left(\cos _{p} \varphi(\tau)\right) \sin _{p} \varphi(\tau) d \tau \\
& +\frac{1}{T_{2} t} \int_{t}^{t+T_{2}} \frac{c(\tau)}{(p-1)}\left|\sin _{p} \varphi(\tau)\right|^{p} d \tau \\
& +\frac{1}{t} \sum_{j=1}^{n} \frac{1}{Q_{j}} \int_{t}^{t+Q_{j}} \frac{\beta_{j}(\tau)}{(p-1) \log _{j}^{2} \tau}\left|\sin _{p} \varphi(\tau)\right|^{p} d \tau \\
& +O\left(\frac{1}{t}\right) .
\end{aligned}
$$

If we add and subtract the below terms in the right side of this equation

$$
\begin{aligned}
& \frac{1}{T_{1} t} \int_{t}^{t+T_{1}} r(\tau)\left|\cos _{p} \theta(t)\right|^{p} d \tau \\
& \quad+\frac{1}{t} \sum_{j=1}^{n} \frac{1}{P_{j}} \int_{t}^{t+P_{j}} \frac{\alpha_{j}(\tau)}{\log _{j}^{2} \tau}\left|\cos _{p} \theta(t)\right|^{p} d \tau \\
& \quad-\frac{1}{\xi t} \int_{t}^{t+\xi} \Phi\left(\cos _{p} \theta(t)\right) \sin _{p} \varphi(\tau) d \tau \\
& \quad+\frac{1}{T_{2} t} \int_{t}^{t+T_{2}} \frac{c(\tau)}{(p-1)}\left|\sin _{p} \theta(t)\right|^{p} d \tau \\
& \quad+\frac{1}{t} \sum_{j=1}^{n} \frac{1}{Q_{j}} \int_{t}^{t+Q_{j}} \frac{\beta_{j}(\tau)}{(p-1) \log _{j}^{2} \tau}\left|\sin _{p} \theta(t)\right|^{p} d \tau
\end{aligned}
$$

we can rewrite this equation as

$$
\begin{aligned}
& \theta^{\prime}(t)=\frac{1}{T_{1} t} \int_{t}^{t+T_{1}} r(\tau)\left|\cos _{p} \theta(t)\right|^{p} d \tau+\frac{1}{t} \sum_{j=1}^{n} \frac{1}{P_{j}} \\
& \cdot \int_{t}^{t+P_{j}} \frac{\alpha_{j}(\tau)}{\log _{j}^{2} \tau}\left|\cos _{p} \theta(t)\right|^{p} d \tau-\frac{1}{\xi t} \\
& \cdot \int_{t}^{t+\xi} \Phi\left(\cos _{p} \theta(t)\right) \sin _{p} \varphi(\tau) d \tau+\frac{1}{T_{2} t} \\
& \cdot \int_{t}^{t+T} \frac{c(\tau)}{(p-1)}\left|\sin _{p} \theta(t)\right|^{p} d \tau+\frac{1}{t} \sum_{j=1}^{n} \frac{1}{Q_{j}} \\
& \cdot \int_{t}^{t+Q_{j}} \frac{\beta_{j}(\tau)}{(p-1) \log _{j}^{2} \tau}\left|\sin _{p} \theta(t)\right|^{p} d \tau+\frac{1}{T_{1} t} \\
& \cdot \int_{t}^{t+T_{1}} r(\tau)\left\{\left|\cos _{p} \varphi(\tau)\right|^{p}-\left|\cos _{p} \theta(t)\right|^{p}\right\} d \tau \\
& \quad+\frac{1}{t} \sum_{j=1}^{n} \frac{1}{P_{j}} \int_{t}^{t+P_{j}} \frac{\alpha_{j}(\tau)}{\log _{j}^{2} \tau}\left\{\left|\cos _{p} \varphi(\tau)\right|^{p}\right.
\end{aligned}
$$

$$
\begin{aligned}
& \left.-\left|\cos _{p} \theta(t)\right|^{p}\right\} d \tau-\frac{1}{\xi t} \\
& \cdot \int_{t}^{t+\xi}\left\{\Phi\left(\cos _{p} \varphi(\tau)\right) \sin _{p} \varphi(\tau)\right. \\
& \left.-\Phi\left(\cos _{p} \theta(t)\right) \sin \theta(t)\right\} d \tau+\frac{1}{T_{2} t} \\
& \cdot \int_{t}^{t+T_{2}} \frac{c(\tau)}{(p-1)}\left\{\left|\sin _{p} \varphi(t)\right|^{p}-\left|\sin _{p} \theta(t)\right|^{p}\right\} d \tau \\
& +\frac{1}{t} \sum_{j=1}^{n} \frac{1}{Q_{j}} \int_{t}^{t+Q_{j}} \frac{\beta_{j}(\tau)}{(p-1) \log _{j}^{2} \tau}\left\{\left|\sin _{p} \varphi(t)\right|^{p}\right. \\
& \left.-\left|\sin _{p} \theta(t)\right|^{p}\right\} d \tau+O\left(\frac{1}{t}\right) .
\end{aligned}
$$
have

And using the half-linear-trigonometric functions, we

$$
\begin{aligned}
& \left.|| \cos _{p} \varphi(\tau)\right|^{p}-\left|\cos _{p} \theta(t)\right|^{p} \mid \\
& \quad \leq p\left|\int_{\theta(t)}^{\varphi(\tau)}\right| \Phi\left(\cos _{p} s\right)\left(\cos _{p} s\right)^{\prime}|d s| \\
& \quad \leq \operatorname{const}|\varphi(\tau)-\theta(t)|, \\
& \left|\Phi\left(\cos _{p} \varphi(\tau)\right) \sin _{p} \varphi(\tau)-\Phi\left(\cos _{p} \theta(t)\right) \sin _{p} \theta(t)\right| \\
& \quad \leq\left|\int_{\theta(t)}^{\varphi(\tau)}\right|\left(\Phi\left(\cos _{p} s\right) \sin _{p} s\right)^{\prime}|d s| \\
& \quad \leq \operatorname{const}|\varphi(\tau)-\theta(t)|,
\end{aligned}
$$$$
\left.|| \sin _{p} \varphi(t)\right|^{p}-\left|\sin _{p} \theta(t)\right|^{p} \mid \leq \text { const }|\varphi(\tau)-\theta(t)| .
$$

By the Mean Value Theorem we can write

$$
\begin{aligned}
\theta(t)= & \varphi_{1}\left(t_{1}\right)+\sum_{j=1}^{n} \varphi_{2_{j}}\left(t_{2_{j}}\right)+\varphi_{3}\left(t_{3}\right)+\varphi_{4}\left(t_{4}\right) \\
& +\sum_{j=1}^{n} \varphi_{5_{j}}\left(t_{5_{j}}\right)
\end{aligned}
$$

for $t_{1} \in\left[t, t+T_{1}\right], t_{2_{j}} \in\left[t, t+P_{j}\right], j=1,2, \ldots, n, t_{3} \in[t, t+\xi]$, $t_{4} \in\left[t, t+T_{2}\right]$, and $t_{5_{j}} \in\left[t, t+Q_{j}\right], j=1,2, \ldots, n$; thus

$$
\begin{aligned}
|\varphi(\tau)-\theta(t)| \leq & \left|\varphi_{1}(\tau)-\varphi_{1}\left(t_{1}\right)\right| \\
& +\left|\sum_{j=1}^{n}\left(\varphi_{2_{j}}(\tau)-\varphi_{2 j}\left(t_{2_{j}}\right)\right)\right| \\
& +\left|\varphi_{3}(\tau)-\varphi_{3}\left(t_{3}\right)\right| \\
& +\left|\varphi_{4}(\tau)-\varphi_{4}\left(t_{4}\right)\right| \\
& +\left|\sum_{j=1}^{n}\left(\varphi_{5_{j}}(\tau)-\varphi_{5 j}\left(t_{5_{j}}\right)\right)\right| .
\end{aligned}
$$


This implies that

$$
\begin{aligned}
|\varphi(\tau)-\theta(t)| & \leq o\left(\frac{1}{t}\right) \\
\varphi(\tau)-\theta(t) & =o(1) \quad \text { as } t \longrightarrow \infty .
\end{aligned}
$$

And using $\stackrel{*}{r}, \stackrel{*}{c}, \stackrel{*}{\alpha}, \stackrel{*}{\beta_{j}}$, and (33), we get

$$
\begin{aligned}
& \theta^{\prime}(t)=\frac{1}{t}\left[\stackrel{*}{r}+\left\{1+O\left(\frac{1}{t \log t}\right)\right\} \sum_{j=1}^{n} \frac{\alpha_{j}^{*}}{\log _{j}^{2} t}\right] \\
& \cdot\left|\cos _{p} \theta(t)\right|^{p} \\
& +\frac{1}{(p-1) t}\left[\stackrel{*}{c}+\left\{1+O\left(\frac{1}{t \log t}\right)\right\} \sum_{j=1}^{n} \frac{\stackrel{\beta}{\beta}_{j}^{*}}{\log _{j}^{2} t}\right] \\
& \cdot\left|\sin _{p} \theta(t)\right|^{p}-\frac{1}{t} \Phi\left(\cos _{p} \theta(t)\right) \sin _{p} \theta(t)+O\left(\frac{1}{t}\right) .
\end{aligned}
$$

The term $O(1 / t)$ can be written as $\left(\left|\cos _{p} \theta\right|^{p}+\left|\sin _{p} \theta\right|^{p}\right) O(1 / t)$; hence we get

$$
\begin{gathered}
\theta^{\prime}(t)=\frac{1}{t}\left[\stackrel{*}{r}+\left[1+O\left(\frac{1}{t \log t}\right)\right] \sum_{j=1}^{n} \frac{\dot{\alpha}_{j}}{\log _{j}^{2} t}\right. \\
\left.+O\left(\frac{1}{t}\right)\right]\left|\cos _{p} \theta(t)\right|^{p}+\frac{1}{(p-1) t}[\stackrel{*}{\mathcal{c}} \\
\left.+\left\{1+O\left(\frac{1}{t \log t}\right)\right\} \sum_{j=1}^{n} \frac{\stackrel{\beta}{\beta}_{j}}{\log _{j}^{2} t}+O\left(\frac{1}{t}\right)\right] \\
\quad \cdot\left|\sin _{p} \theta(t)\right|^{p}-\frac{1}{t} \Phi\left(\cos _{p} \theta(t)\right) \sin _{p} \theta(t) .
\end{gathered}
$$

Now since all the terms of $O(1 / t \log t) / \log _{j}^{2} t$ are $O(1 / t)$ as $t \rightarrow \infty$ for $j=1,2, \ldots, n$, then all these terms are asymptotically less than $o(1) / \log _{n}^{2} t$. Hence we get

$\theta^{\prime}(t)$

$$
\begin{aligned}
= & \frac{1}{t}\left[\stackrel{*}{r}+\sum_{j=1}^{n} \frac{\dot{\alpha}_{j}}{\log _{j}^{2} t}+\frac{o(1)}{\log _{n}^{2} t}\right]\left|\cos _{p} \theta(t)\right|^{p} \\
& +\frac{1}{(p-1) t}\left[\stackrel{*}{\mathcal{c}}+\sum_{j=1}^{n} \frac{\stackrel{*}{\beta}_{j}}{\log _{j}^{2} t}+\frac{o(1)}{\log _{n}^{2} t}\right]\left|\sin _{p} \theta(t)\right|^{p} \\
& -\frac{1}{t} \Phi\left(\cos _{p} \theta(t)\right) \sin _{p} \theta(t) .
\end{aligned}
$$

The main result of this paper is as follows.

Theorem 4. Let $r, c, \alpha_{j}$, and $\beta_{j}, j=1,2, \ldots, n$, are periodic functions which have different $T_{1}, T_{2}, P_{j}$, and $Q_{j}, j=$ $1,2, \ldots, n$, periods, respectively, and $r(t)>0$ in (17). (i) (17) is oscillatory if ${ }^{* *}{ }^{p-1}>\gamma_{p}$ and nonoscillatory if ${ }^{*}{ }^{*}{ }^{-1}<\gamma_{p}$, where ${ }^{*}$ and ${ }^{*}$ are defined in Lemma 3.

(ii) Let $^{* *}{ }^{p-1}=\gamma_{p}$. If there exists $k \in\{2, \ldots, n\}$ such that

$$
\stackrel{*}{*}_{j} \stackrel{*}{r}^{-1}+(p-1) \gamma_{p}{\stackrel{*}{\alpha}{ }_{j}{ }^{-1}-1}^{-1} \mu_{p}, \quad j=1, \ldots, k-1
$$

and ${\stackrel{*}{\beta_{k}}}_{r}^{*} p^{p-1}+(p-1) \gamma_{p} \alpha_{k}^{*} \stackrel{*}{r}^{-1} \neq \mu_{p}$, then (17) is oscillatory if

$$
\stackrel{*}{*}_{k}^{*} \stackrel{p}{r}^{-1}+(p-1) \gamma_{p} \ddot{\alpha}_{k} \stackrel{*}{r}^{-1}>\mu_{p}
$$

and nonoscillatory if

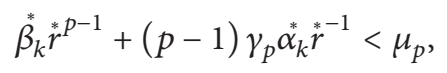

where $\stackrel{*}{\alpha}_{j}$ and $\stackrel{*}{\beta}_{j}, j=1,2, \ldots, n$, are defined in Lemma 3.

Proof. The statement (i) is proved in [10]. It remains to prove the statement (ii) in full generality.

We consider (17); let $x(t)$ be the nontrivial solution of (17) and $\varphi(t)$ is the Prüfer angle of (17) given in (24). Then

$$
\begin{aligned}
\varphi(t)= & \varphi_{1}(t)+\sum_{j=1}^{n} \varphi_{2_{j}}(t)+\varphi_{3}(t)+\varphi_{4}(t)+\sum_{j=1}^{n} \varphi_{5_{j}}(t) \\
& +M
\end{aligned}
$$

is a solution of

$$
\begin{aligned}
\varphi^{\prime}(t)= & \varphi_{1}^{\prime}(t)+\sum_{j=1}^{n} \varphi_{2_{j}}^{\prime}(t)+\varphi_{3}^{\prime}(t)+\varphi_{4}^{\prime}(t) \\
& +\sum_{j=1}^{n} \varphi_{5_{j}}^{\prime}(t)
\end{aligned}
$$

where

$$
\begin{aligned}
\varphi_{1}^{\prime}(t) & =\frac{1}{t} r(t)\left|\cos _{p} \varphi(t)\right|^{p}, \\
\varphi_{2_{j}}^{\prime}(t) & =\frac{\alpha_{j}(t)}{t \log _{j}^{2} t}\left|\cos _{p} \varphi(t)\right|^{p} \quad(j=1,2, \ldots, n), \\
\varphi_{3}^{\prime}(t) & =-\frac{1}{t} \Phi\left(\cos _{p} \varphi(t)\right) \sin _{p} \varphi(t), \\
\varphi_{4}^{\prime}(t) & =\frac{c(t)}{(p-1) t}\left|\sin _{p} \varphi(t)\right|^{p},
\end{aligned}
$$

$$
\varphi_{5_{j}}^{\prime}(t)=\frac{\beta_{j}(t)}{(p-1) t \log _{j}^{2} t}\left|\sin _{p} \varphi(t)\right|^{p}
$$

$$
(j=1,2, \ldots, n) .
$$


By the help of Lemma 3, $\theta(t)$ is a solution of

$\theta^{\prime}(t)$

$$
\begin{aligned}
= & \frac{1}{t}\left[\stackrel{*}{r}+\sum_{j=1}^{n} \frac{\stackrel{\alpha}{j}_{j}}{\log _{j}^{2} t}+\frac{o(1)}{\log _{n}^{2} t}\right]\left|\cos _{p} \theta(t)\right|^{p} \\
& +\frac{1}{(p-1) t}\left[\stackrel{*}{c}+\sum_{j=1}^{n} \frac{\stackrel{\beta}{j}_{j}}{\log _{j}^{2} t}+\frac{o(1)}{\log _{n}^{2} t}\right]\left|\sin _{p} \theta(t)\right|^{p} \\
& -\frac{1}{t} \Phi\left(\cos _{p} \theta(t)\right) \sin _{p} \theta(t),
\end{aligned}
$$

where $\stackrel{*}{r}, \stackrel{*}{c}, \stackrel{*}{\alpha}_{j}$, and $\stackrel{*}{\beta}_{j}, j=1,2, \ldots, n$, are given in Lemma 3 .

This equation is a "Prüfer angle" equation for the following second-order half-linear differential equation

$$
\begin{aligned}
& \left(\left(\stackrel{*}{r}^{+}+\sum_{j=1}^{n} \frac{\stackrel{*}{\alpha}_{j}}{\log _{j}^{2} t}+\frac{o(1)}{\log _{n}^{2} t}\right)^{1-p} \Phi\left(x^{\prime}\right)\right)^{\prime} \\
& +\frac{1}{t^{p}}\left({\stackrel{*}{c}+{ }_{j=1}^{n}}^{n} \sum_{j=1}^{n} \frac{\stackrel{*}{\beta}_{j}}{\log _{j}^{2} t}+\frac{o(1)}{\log _{n}^{2} t}\right) \Phi(x)=0,
\end{aligned}
$$

which is the same as the following equation:

$$
\begin{aligned}
& \left(R(t) \Phi\left(x^{\prime}\right)\right)^{\prime} \\
& \quad+\frac{1}{t^{p}}\left(\stackrel{* *}{c}{ }^{p-1}+\sum_{j=1}^{n} \frac{{ }^{*}{ }_{j} r^{*}{ }^{p-1}}{\log _{j}^{2} t}+\frac{o(1)}{\log _{n}^{2} t}\right) \Phi(x)=0 .
\end{aligned}
$$

Suppose that assumption (ii) of Theorem 4 is satisfied and that (46) holds for $k \in\{1,2, \ldots, n-1\}$. Then (53) is oscillatory as a direct consequence of Theorem 1 . If (46) holds for $k=n$, let $\varepsilon>0$ be so small that still

$$
\stackrel{*}{\beta}_{n} \stackrel{*}{r}^{p-1}-\varepsilon+(p-1) \gamma_{p}\left(\stackrel{*}{r} \stackrel{*}{\alpha}_{j}-\varepsilon\right)>\mu_{p}
$$

and consider the following equation:

$$
\begin{aligned}
& \left(R_{1}(t) \Phi\left(x^{\prime}\right)\right)^{\prime} \\
& \quad+\frac{1}{t^{p}}\left(\stackrel{* *}{c} r^{p-1}+\sum_{j=1}^{n} \frac{\stackrel{*}{\beta}_{j} r^{*} p^{-1}}{\log _{j}^{2} t}+\frac{{\stackrel{*}{\beta_{n}}}_{r}^{*}{ }^{p-1}-\varepsilon}{\log _{n}^{2} t}\right) \Phi(x) \\
& =0,
\end{aligned}
$$

where $R_{1}(t)=\left(1+\sum_{j=1}^{n}\left(\left({ }^{*}{ }_{j} / *\right) / \log _{j}^{2} t\right)+\left({ }^{*}{ }_{n} / \stackrel{*}{r}-\varepsilon\right) / \log _{n}^{2} t\right)^{1-p}$. This equation is a Sturmian minorant for sufficiently large $t$ in (53) and (54) and Theorem 1 implies that this minorant equation is oscillatory and hence (53) is oscillatory as well. This means that the Prüfer angle $\theta(t)$ of the solution of (52) is unbounded and by Lemma 3 the Prüfer angle $\varphi(t)$ of the solution of (17) is unbounded as well. Thus, (17) is oscillatory. A slightly modified argument implies that (17) is nonoscillatory provided that (47) holds.
Corollary 5. If the periods of the functions $r, c, \alpha_{j}$, and $\beta_{j}, j=$ $1,2, \ldots, n$, in (17) coincide with $T$-period, which is given in [6], then our oscillation constants overlap to their oscillation constants and our main result compiles with the result given in [6].

Corollary 6. If there exists a $\operatorname{lcm}\left(T_{1}, T_{2}, P_{j}, Q_{j}\right), j=$ $1,2, \ldots, n$, and the period $T$ which is given in [6] is chosen as $\operatorname{lcm}\left(T_{1}, T_{2}, P_{j}, Q_{j}\right), j=1,2, \ldots, n$, then our oscillation constants overlap to their oscillation constants and our main result compiles with the result given in [6].

Remark 7. If for $j=1,2, \ldots, n \operatorname{lcm}\left(T_{1}, T_{2}, P_{j}, Q_{j}\right)$ is not defined, then only our result can be applied whereas the result given in [6] can not.

Example 8. Consider the nonlinear equation (17) for $p=3$, $r(t)=2+\cos (a x+b),(a, b \in \mathbb{R}), \alpha_{1}(t)=\cos 3 t, \alpha_{2}(t)=\sin 8 t$, $\beta_{1}(t)=\sin 4 t, \beta_{2}(t)=\sin 2 t$, and $c(t)=2+\sin 6 t$. In this case $T_{1}=2 \pi /|a|, P_{1}=2 \pi / 3, P_{2}=\pi / 4, Q_{1}=\pi / 2, Q_{2}=\pi$, and $T_{2}=\pi / 3$ are periods of these functions, respectively. Because of these functions being periodic functions and $r(t)$ positive defined we can use Theorem 4 for all $a \neq 0$ and we obtain

$$
\begin{aligned}
& \stackrel{* * *}{c} p^{p-1}=\left(\frac{|a|}{2 \pi} \int_{0}^{2 \pi /|a|}(2+\cos (a s+b)) d s\right) \\
& \cdot\left(\frac{3}{\pi} \int_{0}^{\pi / 3}(2+\sin 6 s) d s\right)^{3-1}=8 \\
& \gamma_{3}=\left(\frac{3-1}{3}\right)^{3}=\frac{8}{27} \text {. }
\end{aligned}
$$

Thus we get $\stackrel{* *}{c}{ }^{p-1}>\gamma_{3}$ for all $a \neq 0$ and considered equation is oscillatory. Here the important point to note is that while we cannot apply Theorem 2 which is given in [6] for this example if we choose $a=\sqrt{5}$, then $\operatorname{lcm}(2 \pi /|a|, 2 \pi / 3, \pi / 4, \pi / 2, \pi, \pi / 3)$ is not defined, we can apply our Theorem 4 .

\section{Competing Interests}

The authors declare that there is no conflict of interests regarding the publication of this paper.

\section{References}

[1] A. Elbert, "A half-linear second order differential equation," in Qualitative Theory of Differential Equations, Volume I, II, vol. 30 of Colloqula Mathematica Societatis Janos Bolyai, pp. 153-180, North-Holland, Amsterdam, The Netherlands, 1981.

[2] T. Kusano, Y. Naito, and A. Ogata, "Strong oscillation and nonoscillation of quasilinear differential equations of second order," Differential Equations and Dynamical Systems, vol. 2, no. 1, pp. 1-10, 1994.

[3] A. Elbert, "Asymptotic behaviour of autonomous half-linear differential systems on the plane," Studia Scientiarum Mathematicarum Hungarica, vol. 19, pp. 447-464, 1984.

[4] O. Dosly and P. Rehak, Half-Linear Differential Equations, vol. 202 of North-Holland Mathematics Studies, Elsevier Science B.V., Amsterdam, The Netherlands, 2005. 
[5] A. Elbert and A. Schneider, "Perturbations of the half-linear Euler differential equation," Results in Mathematics, vol. 37, no. 1-2, pp. 56-83, 2000.

[6] O. Došlý and H. Funková, "Euler type half-linear differential equation with periodic coefficients," Abstract and Applied Analysis, vol. 2013, Article ID 714263, 6 pages, 2013.

[7] O. Došlý and H. Funková, "Perturbations of half-linear Euler differential equation and transformations of modified Riccati equation," Abstract and Applied Analysis, vol. 2012, Article ID 738472, 19 pages, 2012.

[8] P. Hasil, "Conditional oscillation of half-linear differential equations with periodic coefficients," Archiv der Mathematik, vol. 44, pp. 119-131, 2008.

[9] O. r. Došlý and P. Hasil, "Critical oscillation constant for halflinear differential equations with periodic coefficients," Annali di Matematica Pura ed Applicata, vol. 190, no. 3, pp. 395-408, 2011.

[10] P. Hasil, R. Mařík, and M. Veselý, "Conditional oscillation of half-linear differential equations with coefficients having mean values," Abstract and Applied Analysis, vol. 2014, Article ID 258159, 14 pages, 2014 


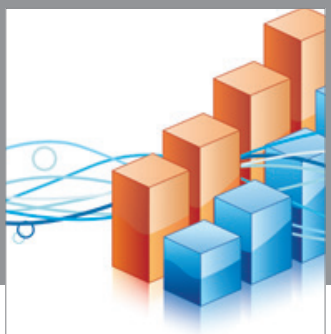

Advances in

Operations Research

vatem alat4

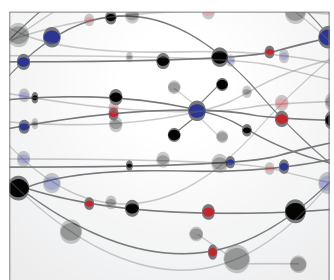

\section{The Scientific} World Journal
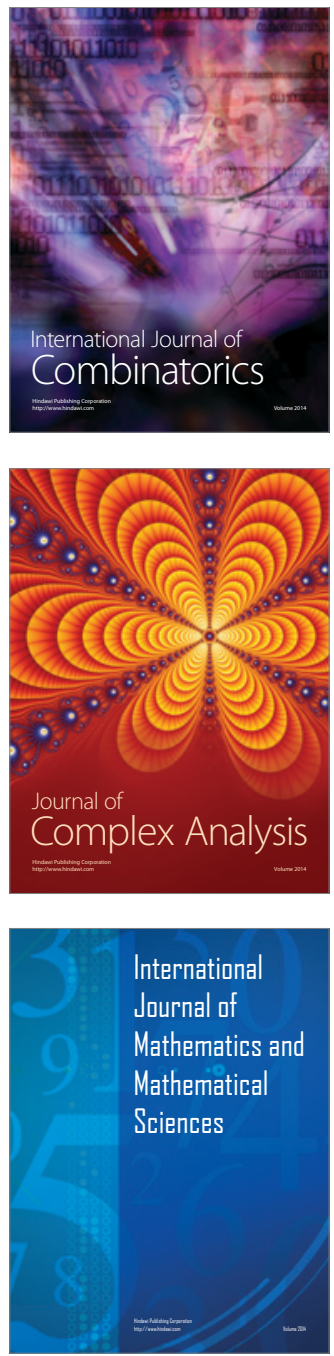
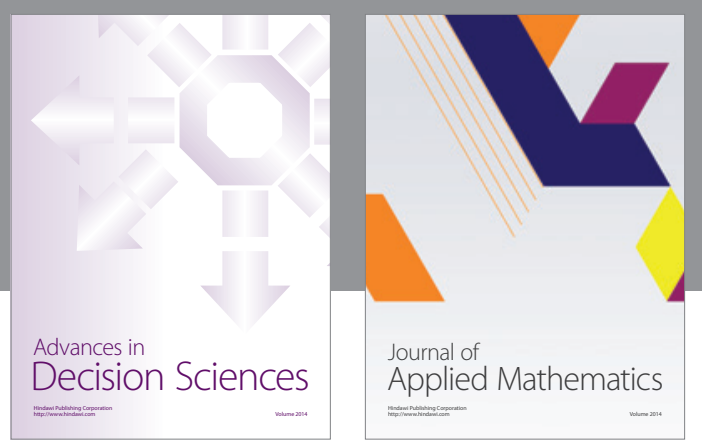

Algebra

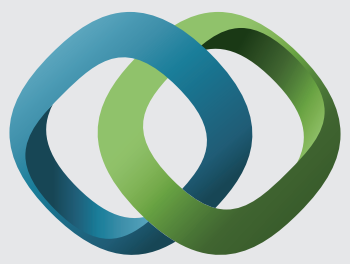

\section{Hindawi}

Submit your manuscripts at

https://www.hindawi.com
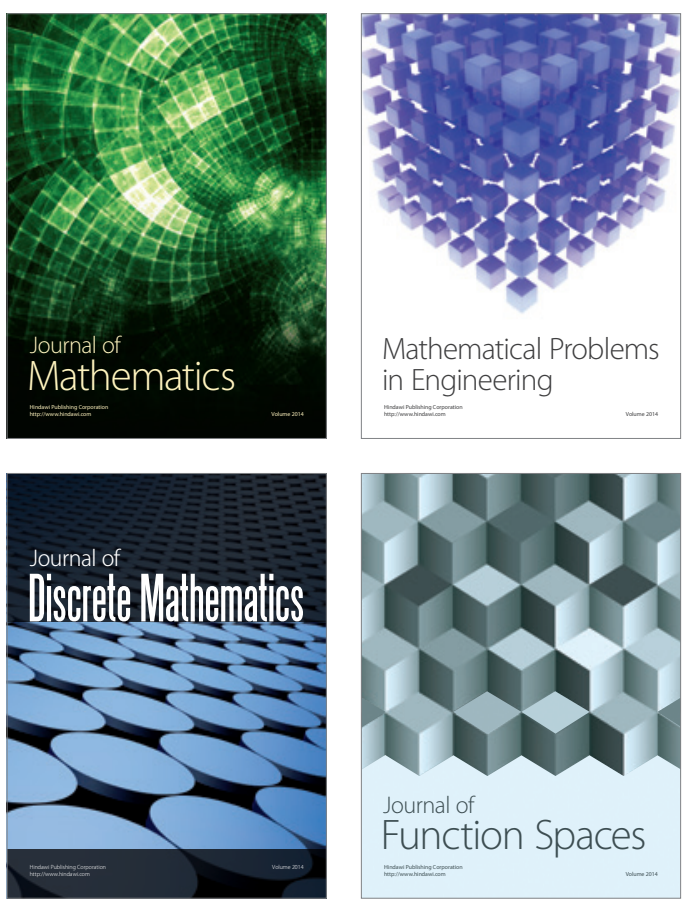

Mathematical Problems in Engineering
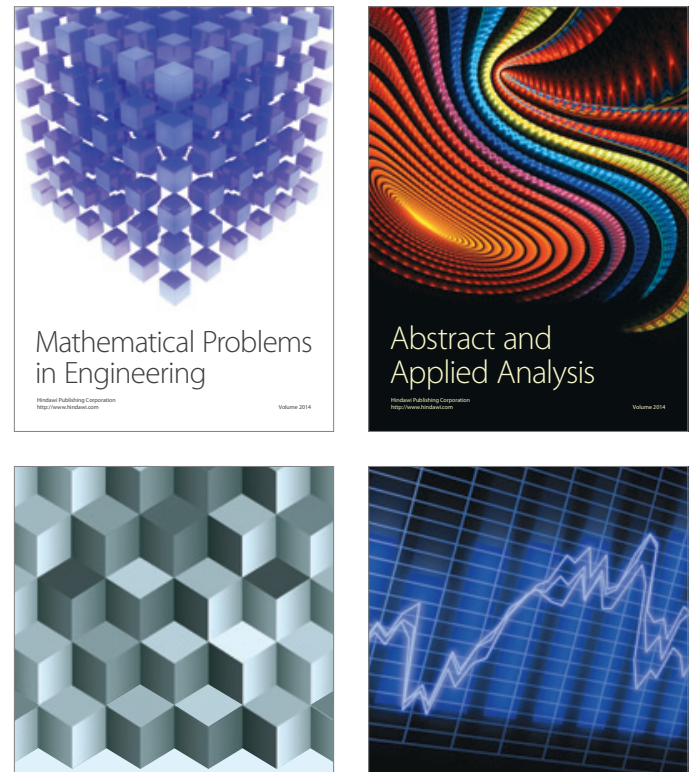

Journal of

Function Spaces

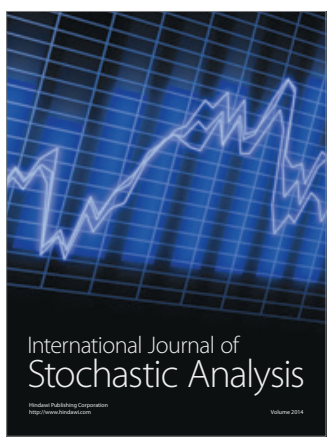

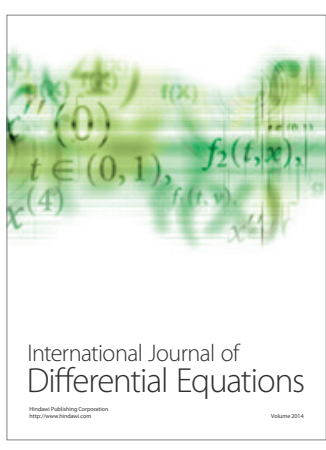
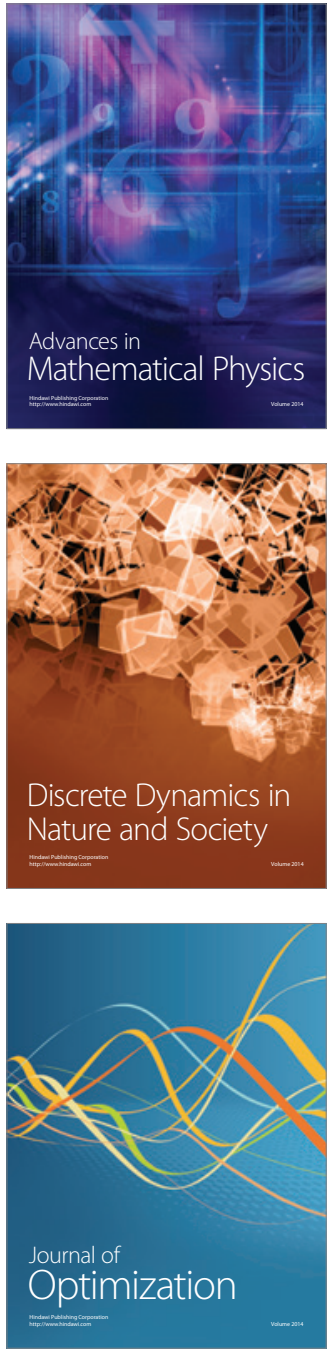